\title{
Crotamine stimulates phagocytic activity by inducing nitric oxide and TNF- $\alpha$ via p38 and NFא-B signaling in RAW 264.7 macrophages
}

\author{
Kyung Jin Lee ${ }^{1}$, Yun Kyu Kim ${ }^{1}$, Martin Krupa ${ }^{2}$, Anh Ngoc Nguyen ${ }^{2}$, Bich Hang Do ${ }^{2}$, Boram Chung ${ }^{2}$, Thi Thu Trang Vu ${ }^{2}$, \\ Song Cheol Kim ${ }^{3}$ \& Han Choe ${ }^{2, *}$ \\ ${ }^{1}$ Department of Convergence Medicine, Asan Institute for Life Sciences, ${ }^{2}$ Department of Physiology and Bio-Medical Institute of \\ Technology, ${ }^{3}$ Department of Surgery, Division of Hepatobilopancreatic Surgery, University of Ulsan College of Medicine, Asan Medical \\ Center, Seoul 05505, Korea
}

Crotamine is a peptide toxin found in the venom of the rattlesnake Crotalus durissus terrificus and has antiproliferative, antimicrobial, and antifungal activities. Herein, we show that crotamine dose-dependently induced macrophage phagocytic and cytostatic activity by the induction of nitric oxide (NO) and tumor necrosis factor-alpha (TNF- $\alpha$ ). Moreover, the crotamineinduced expression of iNOS and TNF- $\alpha$ is mediated through the phosphorylation of p38 and the NF- $\mathrm{KB}$ signaling cascade in macrophages. Notably, pretreatment with SB203580 (a p38specific inhibitor) or BAY 11-7082 (an NF-KB inhibitor) inhibited crotamine-induced NO production and macrophage phagocytic and cytotoxic activity. Our results show for the first time that crotamine stimulates macrophage phagocytic and cytostatic activity by induction of NO and TNF- $\alpha$ via the p38 and NF- $\kappa$ B signaling pathways and suggest that crotamine may be a useful therapeutic agent for the treatment of inflammatory disease. [BMB Reports 2016; 49(3): 185-190]

\section{INTRODUCTION}

Crotamine is a low molecular weight cationic polypeptide that is found in the venom of the South American rattlesnake Crotalus durissus terrificus and possesses several interesting biological activities $(1,2)$. We have successfully produced the toxin as a pure and active form from Escherichia coli (E. coli) (3). The toxin selectively inhibits the three human voltage-gated potassium channels, hKv1.1, hKv1.2, and hKv1.3 (3, 4). It has been reported that hKv1.3 channel blockade enhances the phagocytic function of

*Corresponding author. Tel: +82-2-3010-4292; Fax: +82-2-30108148; E-mail: hchoe@ulsan.ac.kr

http://dx.doi.org/10.5483/BMBRep.2016.49.3.271

Received 29 December 2015, Revised 15 January 2016, Accepted 20 January 2016

Keywords: Crotamine, Macrophage, Nitric oxide, p38, TNF- $\alpha$
RAW264.7 macrophages (5), suggesting that crotamine may enhance macrophage phagocytosis.

Macrophages recognize invading pathogens and are key to mounting an appropriate immune response $(6,7)$. Once activated by stimuli, macrophages upregulate the production of nitric oxide (NO) and various cytokines. Nitric oxide is a free-radical gas that is synthesized by the inducible nitric oxide synthase (iNOS) and mediates a diverse set of functions, including vasodilatation and immunoresponses $(6,8)$. It has also been identified as a major effector molecule in the destruction of cancer cells by activated macrophages (9). In addition, the involvement of NO during macrophage-mediated killing inhibits the proliferation of tumor cells both in vitro and in vivo (10-12).

One of the key cytokines upregulated by activated macrophages is TNF- $\alpha$, which stimulates apoptosis of a wide variety of cells, including tumor cells and cells infected with virus or bacteria (12). The transcription factor NF- $\mathrm{KB}$ is a major survival factor in preventing TNF- $\alpha$ induced apoptosis (13) and its regulation is a key component in several phosphorylation steps in the mitogen-activated protein kinase (MAPK) pathway $(7,14)$. NF- $\kappa B$ plays a critical role in the activation of immune cells by increasing the expression of various cytokines that are essential for immune responses (15).

In this study, we examined how crotamine affects macrophage function. We show for the first time that crotamine enhances macrophage phagocytosis and cytostatic activity, and that it increases NO and TNF- $\alpha$ production by upregulating iNOS and TNF- $\alpha$ expression. Finally, we show that these expression changes are mediated by phosphorylation of $\mathrm{p} 38$ and NF-KB and that direct blockage of these pathways obliterates crotamine's activity.

\section{RESULTS}

Effects of crotamine on phagocytosis and cytostasis of macrophages

The cytotoxic effects of crotamine on RAW 264.7 cells were measured by an MTT assay. Crotamine at $1.5 \mu \mathrm{M}$ did not sig-

ISSN: 1976-670X (electronic edition)

Copyright (C) 2016 by the The Korean Society for Biochemistry and Molecular Biology

(c) This is an open-access article distributed under the terms of the Creative Commons Attribution Non-Commercial License (http://creativecommons.org/licenses/by-nc/4.0) which permits unrestricted non-commercial use, distribution, and reproduction in any medium, provided the original work is properly cited. 
nificantly affect cytotoxicity, as it resulted in $95 \%$ cell viability (Fig. 1A). To determine how crotamine affects the phagocytic activity of macrophages, the uptake of FITC-labeled E. coli particles was compared between crotamine-treated and untreated macrophages. The stimulatory effects of crotamine on macrophage phagocytosis were measured by internalization of the FITC-labeled E. coli particles and were estimated based on the mean fluorescence intensity. Macrophage phagocytosis increased after crotamine treatment in a dose-dependent manner (Fig. 1A). These findings indicate that crotamine increased macrophage phagocytosis, which is one of the functions of macrophages.

To assess other functional activities stimulated by crotamine, the tumor cytostaticity of crotamine-stimulated macrophages was tested against S-180 cells (Fig. 1B). Macrophages showed strong cytostatic activity against the S-180 tumor cells when stimulated with crotamine. However, crotamine at $1.5 \mu \mathrm{M}$ concentrtion did not exhibit any direct killing activity against S-180 cells. These findings indicate that crotamine enhances macrophage cytostaticity against tumor cells.

\section{Effects of crotamine on NO production and TNF- $\alpha$ secretion} To further determine whether crotamine could activate macrophages, we assessed NO production in RAW 264.7 cells. Cells were incubated with crotamine for $24 \mathrm{~h}$, and the NO concentration in culture supernatants was measured using the Griess reaction. With crotamine stimulation, NO synthesis by RAW 264.7 cells was augmented in a dose-dependent manner (Fig. $2 \mathrm{~A})$. Crotamine or LPS in the absence of polymyxin B were previously used as controls (16). Fig. 2B shows that polymyxin B efficiently inhibited NO production induced by LPS, but that it had no effect on crotamine. This finding suggested that the NO produced by crotamine stimulation did not arise from LPS contamination in the crotamine preparation. Moreover, to assess the effects of crotamine on TNF- $\alpha$ production by activated macrophages, we incubated RAW 264.7 cells with increasing concen-
A

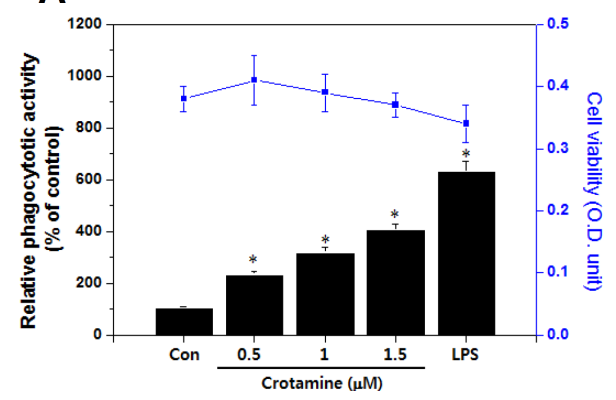

B

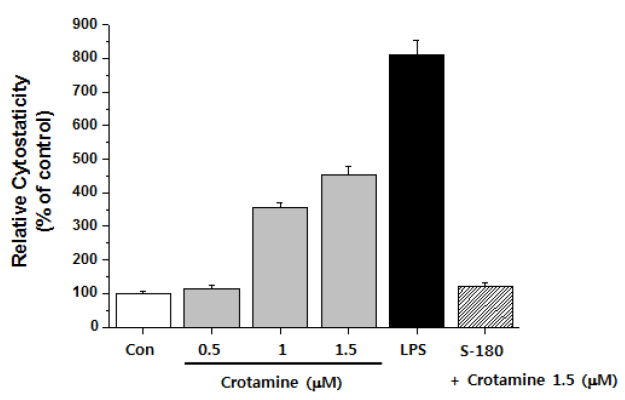

Fig. 1. Effects of crotamine on phagocytosis and cytostasis of macrophages. (A) RAW 264.7 cells were treated with $0.5,1$ or $1.5 \mu \mathrm{M}$ crotamine for $24 \mathrm{~h}$. After FITC labeled-E. coli were added, cells were incubated for $3 \mathrm{~h}$. Supernatants containing unphagocytosed bacteria were removed, and fluorescence was measured using a fluorescence microplate reader. Cell viability was assessed using MTT assays. *Significantly different from control cells $(\mathrm{P}<0.01)$. (B) RAW 264.7 cells were incubated with crotamine or medium alone for $48 \mathrm{~h}$. Cells were then washed twice and further co-cultured with S-180. The cultures were incubated for $24 \mathrm{~h}$, and macrophage cytostaticity was measured according to the MTT method. *Significantly different from control cells $(P<0.01)$.

A

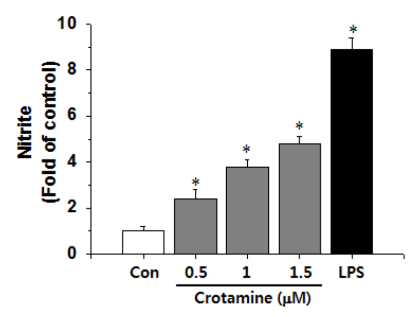

B

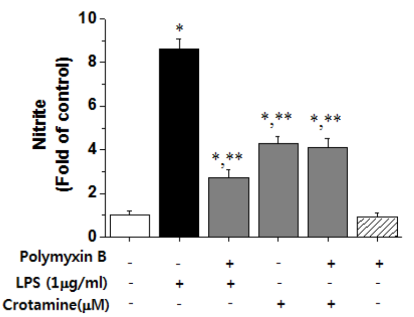

C

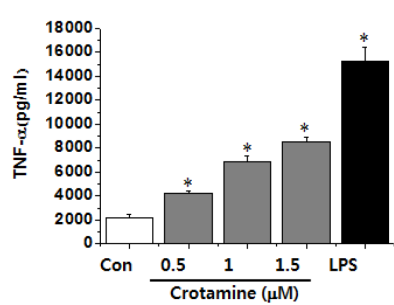

Fig. 2. Effects of crotamine on NO and TNF- $\alpha$ production in macrophages. (A) RAW 264.7 cells were cultured for $24 \mathrm{~h}$ with the indicated concentrations of crotamine, or with LPS $(1 \mu \mathrm{g} / \mathrm{ml})$. Levels of NO production were determined by measuring the accumulation of nitrite in the culture medium. (B) RAW 264.7 cells were cultured with crotamine or LPS in the presence or absence of polymyxin B. Supernatants were harvested $24 \mathrm{~h}$ later and assayed for NO. (C) RAW 264.7 cells were cultured for $6 \mathrm{~h}$ in the presence of media alone or with crotamine. The amounts of TNF- $\alpha$ released into the culture medium were measured by immunoassays. ${ }^{*} \mathrm{P}<0.01$, significantly different from control cells. ${ }^{* *} \mathrm{P}$ $<0.01$, significantly different from LPS-treated cells. 
trations of crotamine. Then, using ELISA, these cytokines were monitored in culture supernatants. Crotamine increased TNF- $\alpha$ secretion in supernatants in a dose-dependent manner (Fig. 2C).

\section{Effects of crotamine on TNF- $\alpha$ and iNOS gene and protein expression in macrophages}

We have shown that crotamine induces the secretion of TNF- $\alpha$ and $\mathrm{NO}$ from macrophages. We now wanted to see if crotamine regulates NO and TNF- $\alpha$ secretion at the mRNA and protein levels. Using a real-time quantitative RT-PCR and western blot assay, we found that crotamine dose-dependently increased both the amount of protein produced (Fig. 3A, B) and the expression levels of TNF- $\alpha$ and iNOS (Fig. 3C, D) in a dose-dependent manner. The control $\beta$-actin was constitutively expressed and was unaffected by crotamine treatment. These results show that crotamine regulates the transcriptional activation of TNF- $\alpha$ and iNOS.

\section{Effects of crotamine on NF- $\mathrm{KB}$ and p38 activation}

The role of crotamine in TNF- $\alpha$ and iNOS gene expression was examined further by investigating the effects of crotamine on $\mathrm{NF}-\mathrm{kB}$ dependent gene expression using a luciferase reporter assay for crotamine-induced NF- $\mathrm{kB}$-dependent luciferase activity (Fig. 4A). We found does-dependent effects on gene expression, which were consistent with the results for $\mathrm{NO}$ and TNF- $\alpha$ protein and mRNA expression levels. Additionally, crotamine increased the levels of the NF-кB subunit p65 in the nucleus (Fig. $4 C)$ by immunoblot analysis. With cell activation, the phosphor-

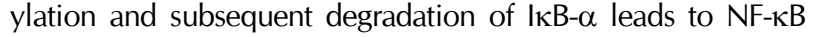
activation. I $\mathrm{kB}-\alpha$ was also rapidly degraded when macrophages were treated with crotamine (Fig. 4B). Anti-lamin B and $\beta$-actin antibodies were used as controls. We can therefore conclude that crotamine-induced NF- $\mathrm{KB}$ activation is a consequence of increased I $\mathrm{k} B-\alpha$ degradation.

The MAPK pathway is a major signaling pathway for cytokines that are associated with macrophages (17). We therefore examined whether the effect of crotamine-induced $\mathrm{NO}$ release and TNF- $\alpha$ production depended on MAPK activation. Western blot analyses were done to detect phosphorylation-specific activation after stimulation with crotamine of the MAP kinases ERK, p38, and JNK. Our results showed that crotamine significantly increases the phosphorylation of p38, but not of JNK or ERK (Fig. 4D).

\section{Effects of crotamine on the induction of NO and TNF-a by p38 and NF-kB mediated signaling}

To further investigate the roles of NF-kB and p38 in NO and macrophage-related TNF- $\alpha$ expression induced by crotamine, we tested the IкB- $\alpha$ kinase inhibitor BAY 11-7082. BAY 11-7082 inhibits the phosphorylation and subsequent degradation of $1 \kappa \mathrm{B}-\alpha$, which is an endogenous NF- $\kappa \mathrm{B}$ inhibitor. Pretreatment of macrophages with BAY 11-7082 reversed NO production (Fig. 4E), phagocytosis (Fig. 4F) and tumor cytotoxicity (Fig. 4G) induced by crotamine. Additionally, SB203580 which is a p38 inhibitor, effectively inhibited NO production as it reversed NO production, phagocytosis, and tumor cytotoxicity (Fig. 4E-G). Together, crotamine-stimulated NO production, phagocytosis and tumor cytotoxicity are mediated by $\mathrm{p} 38$ activation and the NF-KB signaling pathways.
A
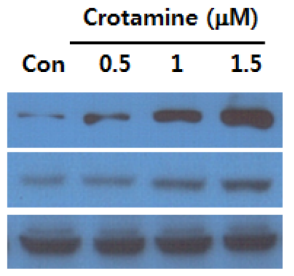

TNF- $\alpha$

iNOS

Actin

B

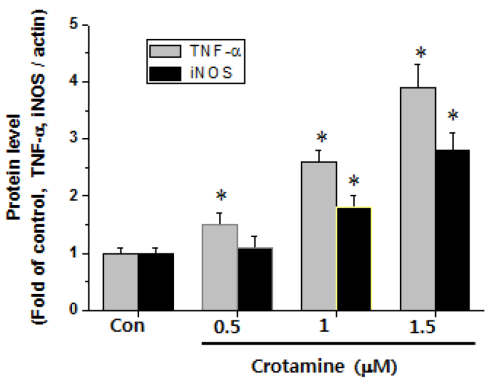

C
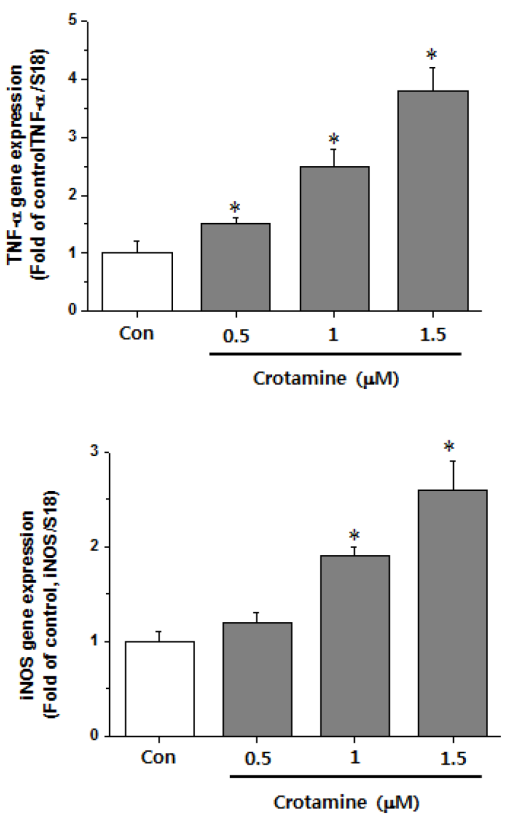

Fig. 3. Effects of crotamine on protein and $\mathrm{mRNA}$ expression levels of TNF- $\alpha$, and iNOS in macrophages. (A) RAW 264.7 cells were treated with crotamine for $18 \mathrm{~h}$ and then lysed. Expression of levels of TNF- $\alpha$ and iNOS were measured by western blotting. (B) After the optical density of protein immunoreactivity was measured and normalized to that of internal control $\beta$-actin. RAW 264.7 cells were treated with crotamine for $3 \mathrm{~h}$ and then were lysed and total RNA was prepared for analysis of TNF- $\alpha(C)$ and iNOS (D) gene expression. PCR amplification of the housekeeping gene, S18, was performed for each sample to assess mRNA expression in exposed cells compared with the expression of unexposed cells at each time point by real-time PCR. *Significantly different from control cells $(\mathrm{P}<$ 0.01). 
A

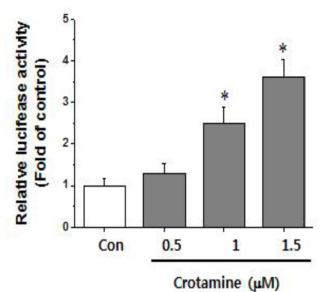

B

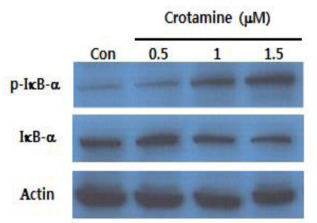

C

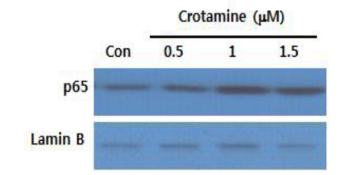

D

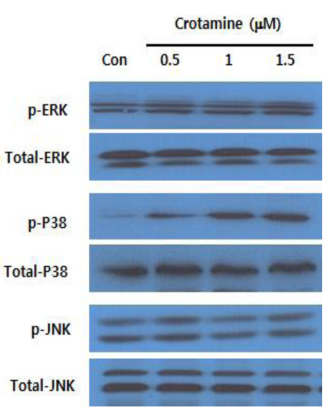

E

F

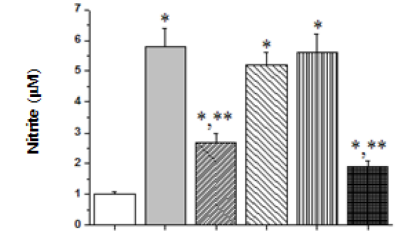

G
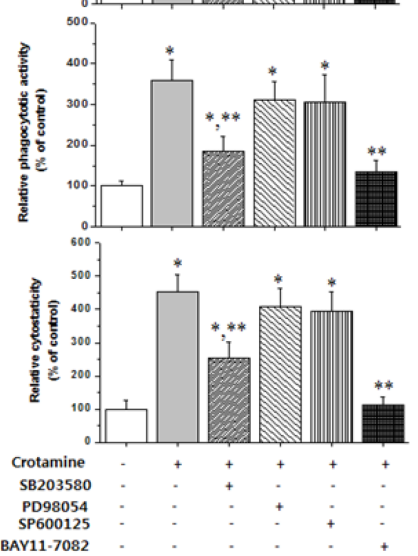

Fig. 4. Crotamine induces NF- $\kappa B$ transactivation and p38 phosphorylation. (A) RAW 264.7 cells were transiently co-transfected with pGL3-NF- $\kappa B-L u c$ and pCMV- $\beta$-gal. After $4 \mathrm{~h}$, cells were treated with the indicated concentrations of crotamine for $18 \mathrm{~h}$. Cells were harvested, and cellular luciferase and $\beta$-galactosidase activities were determined. (B) Crotamine induces the phosphorylation and degradation of IкB- $\alpha$ in macrophages. RAW 264.7 cells were treated with the indicated various concentrations of for 30 min. Western blot analysis was then performed. (C) Cells were treated with crotamine for $20 \mathrm{~min}$, and protein levels of p65 were determined immunochemically using its specific antibody. (D) RAW 264.7 cells were treated with crotamine for $30 \mathrm{~min}$ to assess MAPK expression. Cell extracts were analyzed to assess MAPK activation by western blotting using antibodies specific to phosphorylated and total ERK, p38 and JNK. NF-кB and p38 specific inhibitors reverse crotamine-mediated NO production, phagocytosis and cytostaticity in macrophages. Cells were pretreated with PD98059 (20 $\mu$ M), SB203580 (20 $\mu$ M) and SP600125 $(10 \mu \mathrm{M})$ or BAY $11-7082(10 \mu \mathrm{M})$ for $30 \mathrm{~min}$ and then cultured for $24 \mathrm{~h}$ with crotamine $(1.5 \mu \mathrm{M})$. (E) Levels of NO production were determined by measuring the accumulation of nitrite in culture medium. (F) After FITC labeled-E.coli were added, cells were incubated for 3 h. Supernatants containing unphagocytosed bacteria were removed, and fluorescence was measured using a microplate reader. (G) Macrophage cytostaticity was measured according to the MTT method. $* P<0.01$, significantly different from control. $* * P<0.01$, significantly different from crotamine.

\section{DISCUSSION}

Crotamine is classified as a small basic myotoxin (18) and has specific cytotoxicity in vitro against various neoplastic cells and also was shown to inhibit tumor (19). However, the effects of crotamine on macrophage-related immune functions remain unclear. Therefore, to better understand the effects of crotamine, we investigated its immunostimulatory effects, in particular, its augmentation of macrophage function, including NO production, cytokine production, phagocytosis, and cytostaticity.

The macrophage is an important cell type that is central to cell-mediated and humoral immunity due to its actions as an antigen-presenting, microbicidal, and tumoricidal cell (20). Macrophage phagocytosis represents the initial response to invading microorganisms and enhances the innate immune response. We found that crotamine induces phagocytic activity in RAW 264.7 cells. During phagocytosis, activated macrophages produced $\mathrm{NO}$, which is related to the cytolytic function against various pathogens in macrophages (21).

In this study, we showed that crotamine induced NO and cytokine production in macrophages and that this contributes to the peptide's immunostimulatory activity in RAW264.7 cells.
Moreover, crotamine also enhanced the cytostatic activity of macrophages such as inhibiting the growth of S-180 cells. Cytotoxicity against tumor cells is dependent upon the activation of macrophages, and these mechanisms are closely correlated with the production of several cytokines, such as TNF- $\alpha$ and IL-1 $\beta$. We found that TNF- $\alpha$ levels were increased through increasing level of protein and genes expression. Our results thus elucidate the possible roles of macrophages in the antitumor activities of crotamine.

NF- $\mathrm{BB}$ plays a key role in the transcription of macrophage-related cytokines $(18,22)$. We sought to determine whether crotamine induces TNF- $\alpha$ and iNOS expression in macrophages through the NF- $\mathrm{KB}$ signaling pathway. Using a luciferase assay, we found that crotamine does indeed increase the level of NF- $\kappa B$ activation in macrophages, implicating the NF- $\kappa B$ signaling pathway. In resting macrophages, the NF- $\kappa B$ is localized to the cytosol by interactions with $1 \kappa B$ inhibitory proteins (22). After exposure to pro-inflammatory stimuli, such as NO, TNF- $\alpha$ and $I_{\kappa} \mathrm{B}$ is phosphorylated by IкB kinases $\alpha$ and $\beta$, ubiquitinated, and degraded. The liberated NF-кB dimers (p65/p50) translocate to the nucleus, where transcription of the target genes occurs (23). We showed that crotamine induced the translocation of the 
NF- $\kappa B$ subunit p65 into the nucleus and the subsequent degradation of $I \kappa B-\alpha$. Therefore, crotamine-induced NF- $\kappa B$ activation

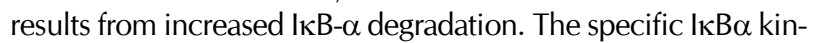
ase inhibitor, BAY 11-7082, inhibited the NO production and phagocytic activity induced by crotamine. BAY 11-7082 inhibits the activation of NF- $\kappa B$ by preventing the phosphorylation and subsequent degradation of $I \kappa B \alpha$. The effects of crotamine were similar to those obtained with BAY 11-7082.

Activated p38 is a key player involved in signaling pathways for macrophage-related cytokine release, phagocytosis and inflammation (24-26). In our study, crotamine significantly increased the phosphorylation of p38, but not ERK and JNK. Our data also showed that specific p38 inhibitors effectively inhibited the NO production and the phagocytic activity induced by crotamine.

Overall, our study shows that crotamine augments RAW 264.7 cells to secrete cytokines and stimulates macrophage phagocytic activity and $\mathrm{NO}$ production. Crotamine increased both, the gene expression and protein levels of TNF- $\alpha$ and iNOS, as well as, increased the phosphorylation and degradation of I $\mathrm{B}-\alpha$, which is required for NF- $\mathrm{B} B$ activation. Thus, our results suggest that crotamine induces macrophage activation and immunostimulatory activity via the NF- $\mathrm{\kappa B}$ signaling pathway, and that crotamine increases the secretion of NO and TNF- $\alpha$ by macrophages, thereby modulating the host's immune response. Crotamine may be useful in the clinic as a peptide therapeutic to activate macrophages.

\section{MATERIALS AND METHODS}

\section{Materials \\ Materials used are listed in Supplementary section S1.}

\section{Preparation of crotamine}

Crotamine was prepared as described previously (3) with minor modifications. Pure crotamine was obtained after applying onto HiTrap SP and HiLoad 26/600 Superdex 200 column with Tris buffer. Finally, the endotoxins were removed by $1 \%$ Triton X-114 (27).

\section{Cell cultures}

The RAW 264.7 cell line was obtained from the ATCC (Bethesda, MD). The cell culture method can be found in Supplementary section S2.

\section{Proliferative activity of macrophages}

RAW 264.7 cells used for the MTT-based assay were measured the according to the manufacturer's instructions. Further details can be found in Supplementary section S3.

\section{Macrophage phagocytosis}

The phagocytosis assay was performed using a commercially available IncuCyte phagocytosis assay kit (Essen Bioscience, Michigan) and were performed according to the manufacturer's instructions. Cells were collected in cold PBS and analyzed using a Microplate-Fluorometer (Molecular Devices, Menlo Park, CA).

\section{Macrophage cytostaticity}

RAW 264.7 cells $\left(5 \times 10^{5}\right.$ cells/well) on 48-well culture plates were incubated with crotamine for $48 \mathrm{~h}$. After washing, cell cytostaticity against tumor cells was assayed by co-culture with $\mathrm{S}-180$. The cultures were incubated for $18 \mathrm{~h}$. Macrophage cytostaticity was measured according to the MTT method. Cytostaticity $(\%)=[1-$ (absorbance at $575 \mathrm{~nm}$ of experiment/absorbance at $575 \mathrm{~nm}$ of control)] $\times 100$.

\section{NO assay}

NO was measured as its stable oxidative metabolite, nitrite $\left(\mathrm{NO}_{x}\right)$, as previously described (28).

\section{Measurement of TNF- $\alpha$ production}

For cytokine immunoassays, cells were cultured for 6 and $24 \mathrm{~h}$ at a density of $5 \times 10^{5}$ cells/well in 48 -well plates. Supernatants were removed at the indicated times, and TNF- $\alpha$ production was quantified by sandwich immunoassays using a protocol supplied by R\&D Systems.

\section{RNA preparation and mRNA analysis by real-time quantitative PCR}

Total RNA was isolated from cells using Trizol (GibcoBRL, Grand Island, NY). Accumulated PCR products were directly detected by monitoring the increase in reporter dye $\left(\mathrm{SYBR}^{\mathbb{R}}\right)$. The expression levels of TNF- $\alpha$ and iNOS in the treated cells were compared to those in control cells at each time point using the comparative cycle threshold $\left(C_{t}\right)$ method $(17,28)$. Primers used are listed in Supplementary Table 1. The quantity of each transcript was calculated as described in the instrument manual and was normalized to the amount of S18, a house-keeping gene.

\section{Transient transfection and luciferase activity assay}

For transient transfections, cells were seeded at $5 \times 10^{5}$ cells/ well in a 48-well plate. The expression vector that contained the $\mathrm{NF}-\kappa \mathrm{B}$ luciferase reporter construct ( $\mathrm{pNF}-\kappa \mathrm{B}-\mathrm{LUC}$, Stratagene, Grand Island, NY) or the empty vector were transfected with 0.5 $\mu \mathrm{L}$ of serum- and antibiotic free LipofectAMINE 2000 reagent (Invitrogen, Carlsbad, CA). After 6 h, the medium was replaced with basal medium. Cells were treated with crotamine for $18 \mathrm{~h}$ and then lysed. Luciferase and $\beta$-galactosidase activities (normalized control) were measured in cellular extracts as described previously (29).

\section{Westem blotting}

Cells were harvested and washed three times with cold phosphate-buffered saline (PBS). Cytoplasmic and nuclear protein fractions were extracted using NE-PER extraction reagents according to the manufacturer's protocol (Pierce Biotechnology, Rockford, IL). 


\section{Statistical analysis}

Statistical methods can be found in Supplementary section S4.

\section{ACKNOWLEDGEMENTS}

This work was supported by Medical Research Center Program (2008-0062286) through the National Research Foundation funded by the Ministry of Science, ICT \& Future Planning, and a grant (2015-307) from the Asan Institute for Life Sciences, Seoul, Korea.

\section{REFERENCES}

1. Kerkis I, Hayashi MA, Prieto da Silva AR et al (2014) State of the art in the studies on crotamine, a cell penetrating peptide from South American rattlesnake. Biomed Res Int 2014, 675985

2. Kerkis I, Silva Fde S, Pereira A, Kerkis A and Radis- Baptista G (2010) Biological versatility of crotamine--a cationic peptide from the venom of a South American rattlesnake. Expert Opin Investig Drugs 19, 1515-1525

3. Vu TT, Jeong B, Yu J et al (2014) Soluble prokaryotic expression and purification of crotamine using an $\mathrm{N}$-terminal maltose-binding protein tag. Toxicon 92, 157-165

4. Peigneur S, Orts DJ, Prieto da Silva AR et al (2012) Crotamine pharmacology revisited: novel insights based on the inhibition of KV channels. Mol Pharmacol 82, 90-96

5. Zhu H, Yan L, Gu J, Hao W and Cao J (2015) Kv1.3 channel blockade enhances the phagocytic function of RAW264.7 macrophages. Sci China Life Sci 58, 867-875

6. Sinder BP, Pettit AR and McCauley LK (2015) Macrophages: Their Emerging Roles in Bone. J Bone Miner Res [Epub ahead of print]

7. Natoli G, Costanzo A, lanni A et al (1997) Activation of SAPK/JNK by TNF receptor 1 through a noncytotoxic TRAF2-dependent pathway. Science 275, 200-203

8. Rodriguez D, Silvera R, Carrio R et al (2013) Tumor microenvironment profoundly modifies functional status of macrophages: peritoneal and tumor-associated macrophages are two very different subpopulations. Cell Immunol 283, $51-60$

9. Teng L, Fu H, Wang M, Deng C and Chen J (2015) Stimulation of RAW264.7 macrophages by sulfated Escherichia coli K5 capsular polysaccharide in vitro. Mol Med Rep 12, 5545-5553

10. Schins RP and Donaldson K (2000) Nuclear factor Kappa- B activation by particles and fibers. Inhal Toxicol 12 Suppl 3, 317-326

11. Calderon C, Huang ZH, Gage DA, Sotomayor EM and Lopez DM (1994) Isolation of a nitric oxide inhibitor from mammary tumor cells and its characterization as phosphatidyl serine. J Exp Med 180, 945-958

12. Farias-Eisner R, Sherman MP, Aeberhard E and Chaudhuri G (1994) Nitric oxide is an important mediator for tumoricidal activity in vivo. Proc Natl Acad Sci U S A 91, 94079411

13. Karin M and Greten FR (2005) NF-kappaB: linking in- flammation and immunity to cancer development and progression. Nat Rev Immunol 5, 749-759

14. Jin X, Wang J, Xia ZM et al (2015) Anti-inflammatory and anti-oxidative activities of paeonol and its metabolites through blocking MAPK/ERK/p38 signaling pathway. Inflammation [Epub ahead of print]

15. Leceta J, Gomariz RP, Martinez C, Abad C, Ganea D and Delgado M (2000) Receptors and transcriptional factors involved in the anti-inflammatory activity of VIP and PACAP. Ann N Y Acad Sci 921, 92-102

16. Sato T, Shoji H and Koga N (2003) Endotoxin adsorption by polymyxin B immobilized fiber column in patients with systemic inflammatory response syndrome: the Japanese experience. Ther Apher Dial 7, 252-258

17. Han EH, Hwang YP, Lee KJ, Jeong TC and Jeong HG (2008) 1-Bromopropane induces macrophage activation via extracellular signal-regulated kinase $1 / 2$ MAPK and NF-kappaB pathways. Cancer Lett 262, 28-36

18. Chang CC and Tseng KH (1978) Effect of crotamine, a toxin of South American rattlesnake venom, on the sodium channel of murine skeletal muscle. Br J Pharmacol 63, 551-559

19. Pereira A, Kerkis A, Hayashi MA et al (2011) Crotamine toxicity and efficacy in mouse models of melanoma. Expert Opin Investig Drugs 20, 1189-1200

20. Cavaillon JM (1994) Cytokines and macrophages. Biomed Pharmacother 48, 445-453

21. Hibbs JB Jr, Taintor RR and Vavrin Z (1987) Macrophage cytotoxicity: role for L-arginine deiminase and imino nitrogen oxidation to nitrite. Science 235, 473-476

22. Ghosh S, May MJ and Kopp EB (1998) NF-kappa B and Rel proteins: evolutionarily conserved mediators of immune responses. Annu Rev Immunol 16, 225-260

23. MacMicking J, Xie QW and Nathan C (1997) Nitric oxide and macrophage function. Annu Rev Immunol 15, 323-350

24. Escolano A, Martinez-Martinez S, Alfranca A et al (2014) Specific calcineurin targeting in macrophages confers resistance to inflammation via MKP-1 and p38. EMBO J 33, 1117-1133

25. Qin Y, Zhou ZW, Pan ST et al (2015) Graphene quantum dots induce apoptosis, autophagy, and inflammatory response via p38 mitogen-activated protein kinase and nuclear factor-kappaB mediated signaling pathways in activated THP-1 macrophages. Toxicology 327, 62-76

26. Arayan LT, Simborio HL, Reyes AW et al (2015) The effects of red ginseng saponin fraction-A (RGSF-A) on phagocytosis and intracellular signaling in Brucella abortus infected RAW 264.7 cells. FEMS Microbiol Lett 362, fnv070

27. Liu S, Tobias R, McClure S, Styba G, Shi Q and Jackowski G (1997) Removal of endotoxin from recombinant protein preparations. Clin Biochem 30, 455-463

28. Lee D, Bae J, Kim YK et al (2013) Inhibitory effects of berberine on lipopolysaccharide-induced inducible nitric oxide synthase and the high-mobility group box 1 release in macrophages. Biochem Biophys Res Commun 431, 506511

29. Bae J, Lee D, Kim YK, Gil M, Lee JY and Lee KJ (2013) Berberine protects 6-hydroxydopamine-induced human dopaminergic neuronal cell death through the induction of heme oxygenase-1. Mol Cells 35, 151-157 